\title{
Nucleus Accumbens Response to Incentive Stimuli Anticipation in Children of Alcoholics: Relationships with Precursive Behavioral Risk and Lifetime Alcohol Use
}

\author{
Wai-Ying Wendy Yau, ${ }^{1,2,3}$ Jon-Kar Zubieta, ${ }^{1,3}$ Barbara J. Weiland, ${ }^{1,2}$ Preeti G. Samudra, ${ }^{1,2}$ Robert A. Zucker, ${ }^{1,2}$ \\ and Mary M. Heitzeg ${ }^{1,2}$ \\ ${ }^{1}$ Department of Psychiatry, ${ }^{2}$ Addiction Research Center, and ${ }^{3}$ Molecular and Behavioral Neuroscience Institute, University of Michigan, Ann Arbor, \\ Michigan 48109
}

Children of alcoholics (COAs) are at elevated risk to develop alcohol and other substance use disorders. The neurobiological underpinnings of this heightened vulnerability are presently not well understood. This study investigated whether, in humans, COAs have different functioning of the mesolimbic reward circuitry beyond previous substance use confounds and examined potential group differences in neural response in relation to alcohol use and behavioral risk. We studied 2018 - to 22-year-old COAs and 20 controls, developmentally well characterized for substance use and selected to match on sex, age, IQ, lifetime substance use and associated problems, and precursive (ages 12-14 years) externalizing behavioral risk. None met criteria for Diagnostic and Statistical Manual of Mental Disorders IV diagnosis. Neural responses to anticipation of reward and loss were assessed using functional magnetic resonance imaging during a monetary incentive delay task. Overall, COAs showed reduced ventral striatum activation during anticipation of monetary reward and loss compared with controls. However, additional analysis revealed that blunted nucleus accumbens (NAcc) response was only observed in COAs who have not demonstrated any problem drinking behavior. In addition, uniquely in COAs, NAcc activation was positively correlated with precursive externalizing risk, as well as current and lifetime alcohol consumption. These findings suggest a multilevel developmental process whereby lower precursive behavioral risk appears protective of later problem alcohol use in COAs, which is further associated with a blunted NAcc response to incentive anticipation, potentially reflecting a resilience mechanism. Moreover, the results suggest that a close association between motivational responses, alcohol consumption, and behavioral risk may underlie addiction vulnerability in COAs.

\section{Introduction}

Alcoholism is a widespread, debilitating disorder that carries high personal and social costs. Individuals transitioning from adolescence to adulthood are especially at risk, because peak 12 month prevalence of alcohol use disorder (AUD) occurs between ages 18 and 23 years (Harford et al., 2005). In addition, parental alcoholism significantly raises risk for offspring alcoholism (Russell, 1990; National Institute on Alcohol Abuse and Alcoholism, 2000), with heritability estimated at 50\% (Goldman et al., 2005). A number of behavioral risk factors for AUD have been established, of which the most validated is childhood externalizing behavior (Cloninger et al., 1988; Englund et al., 2008; Zucker et al., 2008). However, the neurobiological correlates of alcoholism

Received March 18, 2011; revised Dec. 7, 2011; accepted Dec. 14, 2011.

Author contributions: J.-K.Z., R.A.Z., and M.M.H. designed research; W.-Y.W.Y., P.G.S., and M.M.H. performed research; W.-Y.W.Y., B.J.W., and M.M.H. analyzed data; W.-Y.W.Y. and M.M.H. wrote the paper.

This work was supported by K01 DA020088 to MMH, R01 DA027261 to MMH, JKZ and RAZ, T32 AA07477, R01 AA12217 and R37 AA07065 to RAZ and by the Phil F. Jenkins Research Fund (JKZ). The authors would like to acknowledge Tiffany Love, Ph.D. for her valuable contribution to this manuscript.

The authors declare no competing financial interests.

Correspondence should be addressed to Dr. Mary M. Heitzeg, Department of Psychiatry and Addiction Research Center, University of Michigan, 4250 Plymouth Road, Ann Arbor, MI 48109. E-mail: mheitzeg@umich.edu.

DOI:10.1523/JNEUROSCI.1390-11.2012

Copyright $\odot 2012$ the authors $\quad 0270-6474 / 12 / 322544-08 \$ 15.00 / 0$ risk remain largely unclear, and their relationship to behavioral risk is even less well articulated.

Alcohol, like other drugs of abuse, exerts its reinforcing properties by activating the mesolimbic reward circuitry in the brain (Robbins and Everitt, 1999). Modulation of this pathway has been postulated as an important substrate underlying the development of addiction (Blum et al., 2000). Detoxified alcoholics have shown reduced activation in the ventral striatum (VS) during anticipation of monetary incentives relative to controls (Wrase et al., 2007; Beck et al., 2009), whereas another study found differences during reward notification but not anticipation (Bjork et al., 2008a). However, the study of addicts in recovery does not allow for differentiation of biological factors existing before addiction and those resulting from chronic drug exposure.

One approach to studying preexisting risk is to explore whether individuals in high-risk populations can be differentiated in the functional response of reward circuitry that may underlie their vulnerability. The present study investigates the neurobiological signature of familial alcoholism using 18- to 22year old non-addicted offspring recruited from the Michigan Longitudinal Study (MLS) of alcoholic and control families (Zucker et al., 1996). However, even in a non-addicted sample, previous drug exposure may modulate reward circuitry response 


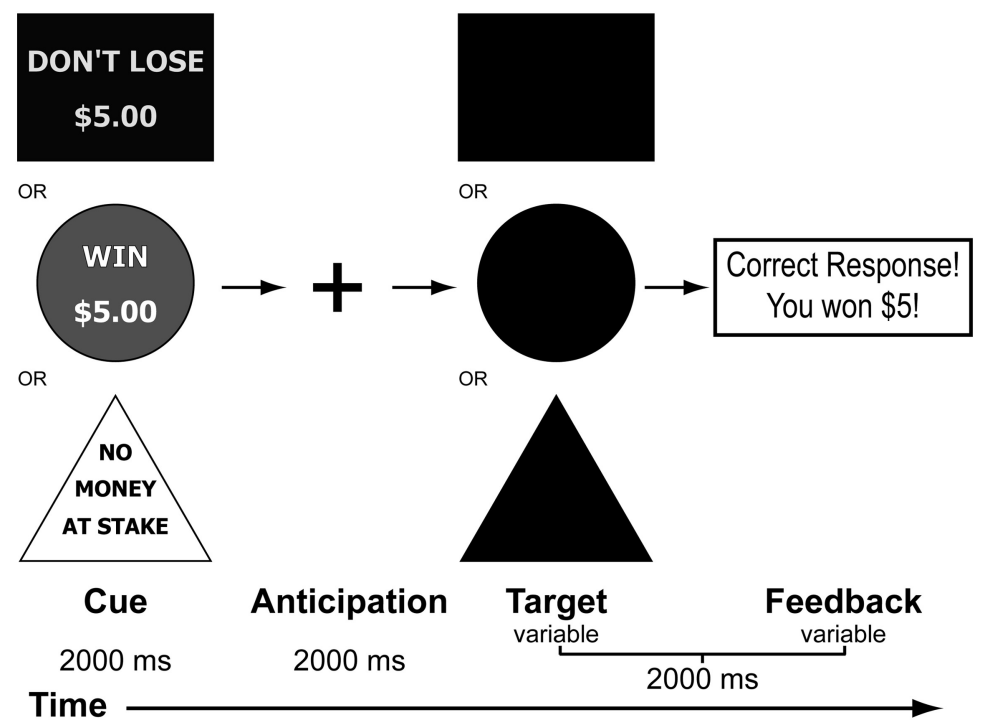

Figure 1. A schematic illustration of the MID task performed by subjects in the fMRI scanner.

Enrollment into the MLS was based on the father's diagnosis, and mother diagnosis was free to vary. Families in which the mother reported drinking during pregnancy or in which the target child exhibited signs of fetal alcohol syndrome (FAS) were excluded from the original ascertainment. Exclusionary FAS characteristics included prenatal or postnatal growth retardation or both, CNS involvement, and characteristic facial dysmorphology (Sokol and Clarren, 1989; Loukas et al., 2001). Control subjects had no parental history of any AUDs. The recruitment strategy and assessment procedures of the MLS have been described in detail previously (Zucker et al., 2000).

The MLS provides long-term characterization of precursive risk beginning in early childhood. From the time of MLS enrollment, all family members are assessed at 3 year intervals with an extensive psychosocial battery of measures assessing temperament, behavioral symptomatology, IQ, school performance, social interaction, etc. During the 11-26 year period, all offspring are also assessed yearly. Given that there were twice as many available

(Boileau et al., 2006; Nocjar and Panksepp, 2007). Children of alcoholics (COAs), as a group, have higher levels of problem drinking and drug use (Sher et al., 1991; Chassin et al., 1999). The longitudinal nature of the ongoing MLS provided early, precursive measures of drinking and drug use, allowing us to minimize this confound by matching the COA group to the control group on levels of previous substance use.

Mesolimbic reward circuitry activation was probed using a modified monetary incentive delay (MID) task (Knutson et al., 2000), in which the anticipation period when participants prepared to make a motor response to attain different reward values was studied. We hypothesized that familial AUD vulnerability would relate to disrupted incentive processing in the nucleus accumbens (NAcc). However, controversy exists regarding the expected direction of this alteration. A hypo-responsive reward system may create a reward-deficiency state that drives compensatory, compulsive drug use (Blum et al., 2000) whereas a hyperresponsive system may bias responses toward immediate rewards, thereby heightening vulnerability (McClure et al., 2004; Hariri et al., 2006). We further investigated the effects of familial risk on these mechanisms as a function of risky alcohol use and explored the relationship between incentive response, early externalizing risk, and alcohol consumption. We hypothesize that NAcc activation during reward anticipation would be related to both variables.

\section{Materials and Methods \\ Participants}

Twenty right-handed COAs (12 males and 8 females; mean age, $20.2 \pm$ 1.2 years; range, $18-22$ years) and 20 controls ( 12 males and 8 females; mean age, $20.1 \pm 1.3$ years; range, $18-22$ years) participated in this study. Subjects were participants in the MLS, an ongoing, prospective community study of families with high levels of parental AUD (two-thirds of sample) and at least one 3 - to 5-year-old male child, along with a contrast sample (one-third of sample) of families of like composition drawn from the same neighborhoods but without a high substance abuse profile (Zucker et al., 1996, 2000). Family history positive subjects had one or both parents with a lifetime diagnosis of AUD, with the majority having at least one parent with an active diagnosis during the life of the child. Parental diagnosis was based on Diagnostic and Statistical Manual of Mental Disorders IV criteria and established via multiple face-to-face diagnostic assessments of the parents over the course of the youth's life. offspring from the MLS in the COA sample, we were able to select COAs for this study to match the controls across sex, IQ, levels of alcohol and other drug consumption, alcohol and drug problems, and early adolescent externalizing behavior problems.

Exclusion criteria for subjects in the present study include the following: any neurological, acute, uncorrected, or chronic medical illness; any Axis I psychiatric or developmental disorders; any current or recent (within 6 months) treatment with centrally active medications; a history of psychosis or schizophrenia in first-degree relatives; and a positive urine drug screen on the day of the study. Written informed consent, approved by the University of Michigan Medical School Institutional Review Board, was obtained before the study.

\section{Measures}

fMRI paradigm. Brain response during anticipation of incentive stimuli was probed in an fMRI experiment using a modified version of the MID task published by Knutson et al. (2000). A schematic of the modified paradigm is presented in Figure 1. Each $6 \mathrm{~s}$ trial consisted of four events. First, subjects were presented with an incentive cue $(2000 \mathrm{~ms})$ of five possible values (gain of $\$ 0.2, \$ 5.0$; loss of $\$ 0.2, \$ 5.0$; or no change $\$ 0$ ). This was followed by a $2000 \mathrm{~ms}$ anticipation delay. Next, a target appeared for a variable length of time (200-300 ms), during which subjects made a button-press response in an attempt to gain or avoid losing the money. Subjects were instructed to respond to neutral targets despite the lack of incentive value. A feedback message then informed them of the trial outcome. The incentive trials were presented contiguously in a pseudorandom order. Two runs of the task were performed, each lasting $5 \mathrm{~min}$. A total of 20 trials per each condition were recorded. The duration of the response target was calculated based on the individual subject's reaction time (RT) during a practice session before scanning. The allotted duration was calibrated such that the overall success rate was $\sim 60 \%$. Participants were paid a fixed rate to participate in the study and additionally received any money they won during the task.

Alcohol and substance use history and current involvement. Current alcohol and cigarette consumption information (drinks consumed per week and cigarettes smoked per day over the past month) was collected through telephone interview during study recruitment. Drinking and drug use was measured longitudinally using the Drinking and Drug History Form (Zucker, 1991) as part of the MLS protocol. Binge drinking measures included the following: (1) the number of days in the past 6 months (reported at most recent MLS annual assessment) during which the subject had consumed five or more (four or more in the case of females) standard drinks of beer, wine, or liquor; and (2) the maximum number of drinks consumed in $24 \mathrm{~h}$ (reported at most recent MLS annual 
Table 1. Means (SDs) of critical study characteristics for controls and COAs

\begin{tabular}{lccc}
\hline & & & $t$ test \\
& Control $(n)$ & COA $(n)$ & $p$ value \\
\hline Males:Females & $12 / 8$ & $12 / 8$ & \\
Age (years) & $20.1(1.3)$ & $20.2(1.2)$ & 0.7 \\
IQ & $113.2(8.0)$ & $110.9(12.4)$ & 0.5 \\
Cigarettes per day (time of scan) & $1.5(4.7)$ & $2.2(5.0)$ & 0.6 \\
Drinks per week (time of scan) & $5.9(7.2)$ & $5.2(7.3)$ & 0.7 \\
Binge drinking days (past 6 months) & $37.9(54.9)$ & $48.0(76.0)$ & 0.6 \\
Max drinks in 24 h & $8.4(8.7)$ & $9.3(9.4)$ & 0.8 \\
Lifetime drink volume (number of drinks) & $702.5(1067)$ & $788.5(1036)$ & 0.8 \\
Number of never drinkers & 4 & 4 & \\
Number of alcohol problems & $3.8(5.6)$ & $5.2(5.7)$ & 0.4 \\
Number of drug problems & $0.3(1.0)$ & $0.6(1.1)$ & 0.4 \\
Freq of marijuana use (past 12 months) & $1.8(2.9)$ & $2.2(3.0)$ & 0.6 \\
Number of illicit drugs ever used & $1.3(2.4)$ & $1.3(1.3)$ & 1.0 \\
Mother/father/both (alcohol dependence) & $0 / 0 / 0$ & $10 / 15 / 6$ & \\
Mother/father/both (alcohol abuse) & $0 / 0 / 0$ & $6 / 5 / 1$ & \\
Mother/father/both (alcohol dependence or abuse) & $0 / 0 / 0$ & $12 / 17 / 9$ & \\
Mother/father/both (abused other drugs) & $1 / 2 / 0$ & $8 / 9 / 5$ & \\
Externalizing T score (age 12-14 years) & $47.8(10.1)$ & $46.5(8.9)$ & 0.8 \\
Internalizing T score (age 12-14 years) & $46.3(8.8)$ & $47.1(9.4)$ & 0.7 \\
\hline
\end{tabular}

assessment). Participants were also asked how many days they drank in the past 6 months and the 6 months before that, and, on a day when they were drinking, how many drinks they usually had in 24 h over the past 6 months and the 6 months before that. Lifetime drink volume was calculated from these data collected annually since age 11 years as the total number of drinks consumed. Alcohol problem and drug problem scores were the numbers of drinking or drug-related problems (from a possible 37 and 22 items, respectively) ever reported by the subject since the age of 11 years. Marijuana use is defined as the number of occasions the participants reported using marijuana or hashish in the past 12 months reported at the most recent MLS assessment. Number of illicit drugs used is defined as the total number of illicit drugs the participant ever reported using during annual assessments since age of 11 years.

Early adolescent behavioral problems. Externalizing behavior problems were assessed with the Youth Self-Report (YSR) as part of the ongoing MLS. The YSR (Achenbach, 1991), yields scores on eight narrow-band subscales and two broad-band subscales (externalizing and internalizing behavior) and was completed by each participant when they were between 12 and 14 years old. We focused on this age range because of our interest in how earlier behavioral risk relates to later outcome- both level of drinking and responsivity of reward circuitry. Furthermore, YSR data at this age provide a measure of behavior problems evident before significant drinking and drug use and, for many, before any drug involvement. In the COA group, one participant $(5 \%)$ had an externalizing problems score in the borderline clinical range. In the control group, one participant scored in the borderline clinical range and one in the clinical range.

Family severity index. This was calculated based on mother and father diagnoses of alcohol abuse or dependence and of other drug abuse (Table 1). A value of 1 was assigned for each parent with an abuse diagnosis of alcohol or of other drugs; a value of 1.5 was assigned for each parent with an alcohol dependence diagnosis (no other drug dependence criteria were met). This resulted in a possible range of values for COAs of 1 (one parent with alcohol abuse) to 5 (both parents with alcohol dependence and other drug abuse). The actual range was 1.5-5 (mean \pm $\mathrm{SD}, 2.9 \pm 1.3)$.

\section{fMRI data acquisition}

Whole-brain blood oxygen level-dependent functional images were acquired on a 3.0 tesla GE Signa scanner (GE Healthcare) using a T2* weighted single-shot combined spiral in/out sequence (Glover and Law, 2001) with the following imaging parameters: repetition time (TR), 2000 $\mathrm{ms}$; echo time (TE), $30 \mathrm{~ms}$; flip angle, $90^{\circ}$; field of view (FOV), $200 \mathrm{~mm}$; matrix size, $64 \times 64$; in-plane resolution, $3.12 \times 3.12 \mathrm{~mm}$; and slice thickness, $4 \mathrm{~mm}$. A high-resolution anatomical T1 scan was obtained for spatial normalization [three-dimensional spoiled gradient recalled echo (3-DSPGR); TR, 25 ms; minimum TE; FOV, 25 cm; $256 \times 256$ matrix; slice thickness, $1.4 \mathrm{~mm}$ ]. Participant motion was minimized with the use of foam pads placed around the head along with a forehead strap. In addition, the importance of keeping as still as possible was emphasized during the Informed Consent process and at scanner entry.

\section{Data analysis}

fMRI task performance analysis. RT and success rate for each incentive condition were calculated. Mix-effects ANOVA (valence $\times$ amount $\times$ group) were used to assess performance differences between groups, followed by post hoc pairwise comparisons to determine the source of significant interactions.

Functional data preprocessing. Functional images were reconstructed using an iterative algorithm (Sutton et al., 2003; Fessler et al., 2005), which is more robust against image distortions caused by off-resonance effects than conventional methods. Subject head motion and sliceacquisition timing were corrected using the FSL 4.0 analysis tools library (Analysis Group, Functional MRI of the Brain, Oxford, UK) (Jenkinson et al., 2002). Analysis of estimated motion parameters confirmed that overall head motion within each run did not exceed $2 \mathrm{~mm}$ translation or $2^{\circ}$ rotation in any direction. All remaining image processing was completed using statistical parametric mapping SPM2 package (Wellcome Institute of Cognitive Neurology, London, UK). Functional images were spatially normalized to a standard stereotactic space as defined by the Montreal Neurological Institute (MNI). A $6 \mathrm{~mm}$ fullwidth half-maximum Gaussian spatial smoothing kernel was applied to improve signal-to-noise ratio and to account for individual differences in anatomy.

Individual subject statistical maps. Individual analysis was completed using a general linear model. Five regressors of interest (anticipation of win $\$ 0.2$, win $\$ 5.0$, lose $\$ 0.2$, lose $\$ 5.0$, and neutral $\$ 0$ ) were convolved with the canonical hemodynamic response function. Motion parameters were modeled as nuisance regressors to remove residual motion artifacts. Scanner drift and other low-frequency noise were removed from the image time series using a $128 \mathrm{~s}$ high-pass filter. Two contrasts of interest, anticipation of reward ( $\$ 0.2$ and $\$ 5.0$ combined) minus neutral incentive and anticipation of loss ( $\$ 0.2$ and $\$ 5.0$ combined) minus neutral incentive, were calculated for second-level group analyses. Because the present study only modeled the anticipatory period between cue presentation and motor responding, all subsequent reference to incentive activation refers to activity during the anticipation of reward or loss.

Voxel-by-voxel group analyses. Group statistical maps for reward and loss anticipation versus neutral were calculated using one sample $t$ tests. Independent-sample $t$ tests were used to identify regions in which brain activation differed between groups. Statistical significance was established at false discovery rate (FDR)-corrected $p<0.05$ (Genovese et al., 2002). For the a priori hypothesized region (NAcc), statistical significance was reported after small-volume correction (SVC) using anatomical masks described below.

Volume-of-interest analyses. To further characterize the NAcc response to incentive stimuli while avoiding circularity of inference (Kriegeskorte et al., 2009), we performed anatomical volume-of-interest (VOI) analyses. Anatomical masks for the NAcc were created as specified by Bjork et al. (2008a). The published Talairach coordinates mapped to [-10, 13, $-8 ; 11,13,-8]$ in MNI space (Lacadie et al., 2008). Five-mm-diameter spherical masks were created using MarsBaR region-of-interest toolbox (Brett et al., 2002). Figure $3 A$ illustrates the location of the mask at the ventromesial intersection of caudate and putamen (Bjork et al., 2008a). Masks were individually repositioned up to $1 \mathrm{~mm}$ to ensure accurate placement. Visual inspection of each subject's data confirmed that masks were accurately placed on the NAcc. Effect sizes in the VOI for activation during each incentive condition (anticipation of win $\$ 0.2$, win $\$ 5.0$, lose $\$ 0.2$, lose $\$ 5.0$ ) over neutral condition (anticipation of $\$ 0$ ) were calculated from respective contrast images using MarsBaR region-of-interest toolbox.

The following analyses were performed on these VOI data. (1) Hemisphere $X$ valence $\times$ amount $\times$ group mixed-effects ANOVA was performed, followed by post hoc pairwise comparisons to determine the 


\begin{tabular}{|c|c|c|c|c|c|c|}
\hline \multirow[b]{2}{*}{ Brain region } & \multicolumn{6}{|c|}{ MNI coordinates } \\
\hline & Hemisphere & $x$ & $y$ & $z$ & Peak $t$ value & $p$ (FDR-corrected) \\
\hline \multicolumn{7}{|l|}{ Controls } \\
\hline \multirow[t]{2}{*}{ VS } & Left & -12 & 8 & -10 & 9.31 & 0.001 \\
\hline & Right & 10 & 10 & -6 & 8.47 & 0.001 \\
\hline \multirow[t]{2}{*}{ Thalamus } & Mesial & 2 & -6 & 4 & 6.87 & 0.001 \\
\hline & Left & -14 & -6 & 10 & 3.84 & 0.02 \\
\hline Caudate & Right & 18 & 6 & 18 & 4.11 & 0.02 \\
\hline Cingulate & Right & 4 & -4 & 32 & 3.94 & 0.02 \\
\hline \multicolumn{7}{|l|}{$\mathrm{COA}$} \\
\hline \multirow[t]{2}{*}{ VS } & Left & -14 & 10 & -12 & 7.4 & 0.001 \\
\hline & Right & 14 & 8 & -14 & 6.5 & 0.001 \\
\hline Thalamus & Mesial & 2 & -6 & 6 & 7.93 & 0.001 \\
\hline \multirow[t]{2}{*}{ Caudate } & Left & -18 & 0 & 22 & 6.13 & 0.002 \\
\hline & Right & 18 & 2 & 20 & 3.53 & 0.03 \\
\hline \multirow{2}{*}{$\begin{array}{l}\text { MFG-orbital } \\
\text { Insula }\end{array}$} & Right & 22 & 46 & -22 & 5.06 & 0.004 \\
\hline & Right & 32 & 24 & 4 & 3.33 & 0.04 \\
\hline
\end{tabular}

source of significant interactions. (2) Correlations with externalizing behavior scores and current (drinks per week) and lifetime (lifetime drink volume) alcohol consumption were explored across the entire sample $(n=40)$ and within each group. Spearman's rank correlation coefficient was used for current and lifetime alcohol consumption variables due to non-normal distribution (Shapiro-Wilk's test, $p=0.00$ ). Fisher's $Z$-transformation was used to determine differences in correlations between groups. Bonferroni's correction was used to correct for multiple comparisons $(0.05 / 8=p<0.0063)$. (3) ANOVAs were conducted to investigate the effect of risky drinking on NAcc activation and possible interactions with familial risk. Taking into account both consumption and problem use factors, participants were classified as lifetime low-risk versus high-risk drinkers. Low-risk drinkers had never engaged in binge drinking and reported at or below the group median in all of the following three measures: drinks per week consumed at time of scan (2), lifetime drink volume (191), and number of alcohol problems (2.4).

\section{Results}

\section{fMRI task performance}

Because it was the objective of the paradigm to individually calibrate task difficulty to achieve comparable performances, the success rate for each condition did not differ between groups (all $p$ values $>0.43)$. Because of a software error, only RTs to correct trials were recorded. The group $\times$ valence $\times$ amount ANOVA revealed a main effect of amount on $\mathrm{RT}(\$ 0.20 \mathrm{RT}>\$ 5 \mathrm{RT}$; $F_{(1,38)}=6.3, p=0.02$ ), a trend for a main effect of valence (loss $\mathrm{RT}>$ reward RT; $\left.F_{(1,38)}=3.1, p=0.09\right)$, and a significant interaction between valence and amount $\left(F_{(1,38)}=4.8, p=\right.$ $0.03)$. Post hoc comparisons revealed a significant effect of valence for $\$ 5$ trials ( $\$ 5$ loss $\mathrm{RT}>\$ 5$ reward RT; $p=0.02$ ) but not for $\$ 0.20$ trials $(p=0.56)$ and a significant effect of amount for reward trials ( $\$ 0.20$ reward $\mathrm{RT}>\$ 5$ reward RT; $p=0.001)$ but not for loss trials $(p=0.95)$.

\section{Whole-brain voxel-by-voxel group analyses}

Anticipation of monetary reward versus no incentive

Significant regional activations for each group are summarized in Table 2. Anticipation of reward activated bilateral VS, including the NAcc, in both control subjects and COAs (Fig. 2, top row). Because the overall task effects of the MID paradigm have been extensively characterized and are replicated by the current results (Knutson et al., 2001; Bjork et al., 2004, 2008b; Wrase et al., 2007; Carter et al., 2009), this report will focus instead on novel findings of group differences. Independent-sample $t$ test revealed that COAs had significantly lower activation in the right NAcc com- pared with controls (MNI coordinates $x, y, z=14,12,-8$; $t=$ 3.3, FDR-corrected $p=0.02$ after SVC) (Fig. 2, top row). COAs did not show greater activation over controls in any region.

Anticipation of monetary loss versus no incentive

Activated regions for each group are summarized in Table 3. Anticipation of loss activated bilateral VS in both control subjects and COAs. Independent-sample $t$ test showed that COAs had significantly reduced activation in NAcc (MNI coordinates $x, y$, $z=14,10,-6 ; t=3.27$, FDR-corrected $p=0.04$ after SVC) compared with controls (Fig. 2, bottom row). No areas of increased activation in COAs with respect to controls were observed.

\section{NAcc VOI}

Hemisphere $\times$ valence $\times$ amount $\times$ group ANOVA revealed expected main effects of valence $\left(F_{(1,38)}=62.0, p<0.001\right.$; reward $>$ loss $)$ and amount $\left(F_{(1,38)}=31.1, p<0.001 ; \$ 5>\$ 0.2\right)$. There was also a valence $\times$ hemisphere interaction $\left(F_{(1,38)}=5.5\right.$, $p=0.03)$, in which the significant effect of valence was greater in left compared with right hemisphere. A trend for group $\times$ valence interaction was also found $\left(F_{(1,38)}=3.5, p=0.07\right)$. Post hoc comparisons revealed a trend effect of family history in reward trials (COA reward $<$ control reward, $p=0.07$ ) but no such effect in loss-avoidance trials. Because there were no main effect of hemisphere $(p>0.96)$ and no interaction between hemisphere and group $(p>0.1)$, activation in right and left NAcc were combined for remaining VOI analyses.

\section{VOI correlation analyses}

Correlation analyses were conducted with averaged left and right NAcc activation to anticipation of reward and anticipation of loss (\$0.20 and \$5 combined for each). Results are summarized in Table 4 . Across the entire sample $(n=40)$, externalizing problems at ages 12-14 years was positively associated with both current and lifetime alcohol consumption (Table 4). Also, there was a positive correlation between NAcc activation during reward and loss (trend level, $p=0.06$ ) with current alcohol consumption (drinks per week) but not with lifetime drink volume or externalizing problems at ages 12-14 years.

Given our interest in the possibility of different relationships for COAs and controls, correlations were further explored in these groups separately and revealed much different findings between the groups. COAs showed strong positive correlations among current drinks per week, lifetime alcohol volume, precursive externalizing risk, and NAcc activation to both reward and loss anticipation. Conversely, controls only showed a trend positive correlation between current drinks per week and externalizing problems, and a negative relationship was found between externalizing problems and NAcc activation. Comparison of correlation coefficients using Fisher's $Z$ transformation revealed significantly stronger positive correlation between variables in COAs compared with controls (Table 4). The majority of significant relationships survived correction for multiple comparisons. Figure $3 B$ illustrates the opposing correlations between NAcc activation and early externalizing problems in COAs and controls.

In COAs, the family alcoholism severity index did not correlate with NAcc activation (reward, $r=-0.18, p=0.44$; loss, $r=$ $-0.06, p=0.81$ ), externalizing behavior problems, or current or lifetime drinking (all $p$ values $>0.2$ ).

\section{Family history $\times$ risky alcohol use ANOVA}

The relationship between family history and risky alcohol use on NAcc responding were further explored using an ANOVA design, 


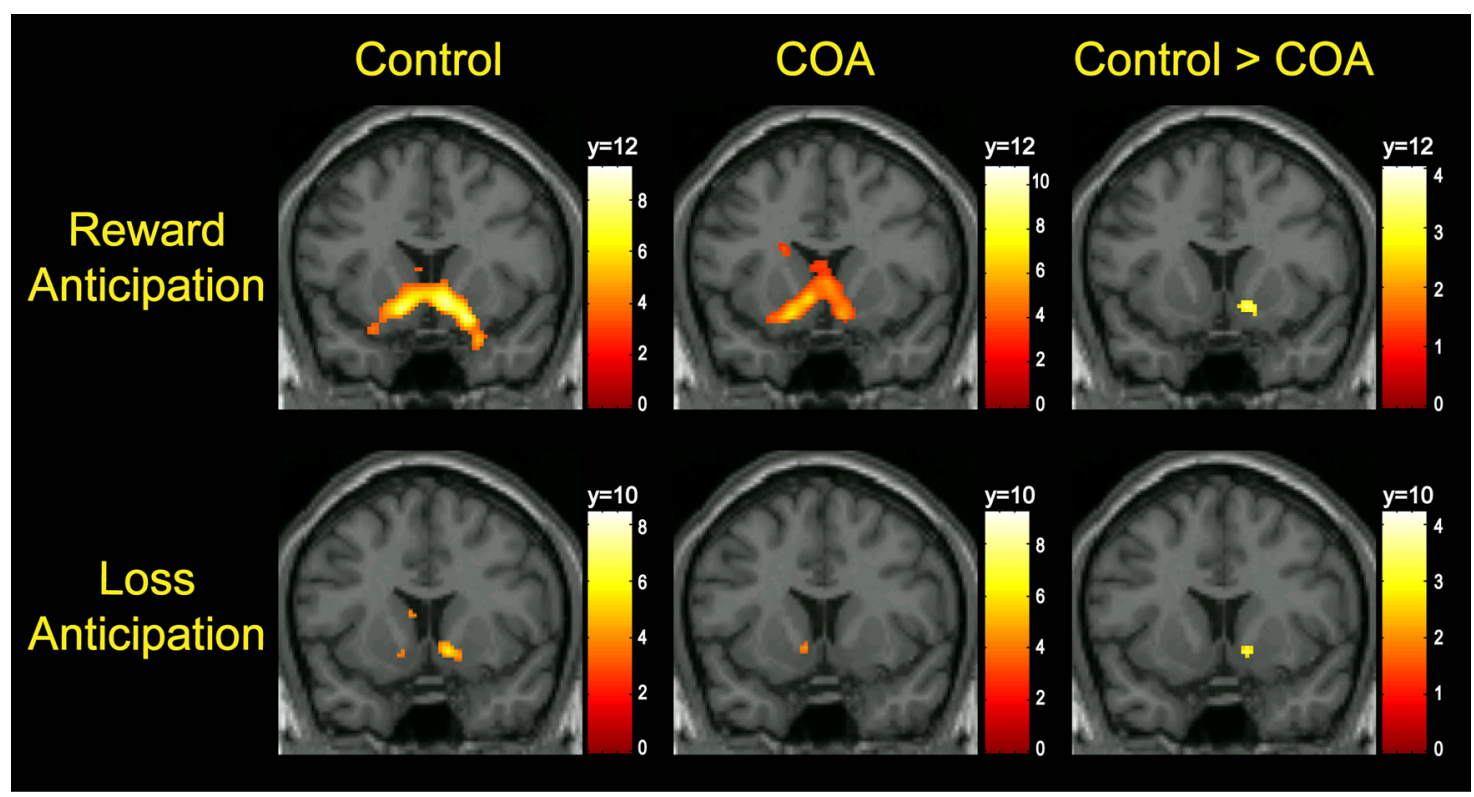

Figure 2. Top row, Activation in VS during reward anticipation in control and COA groups and result of independent-sample $t$ test of control $>$ COA. Bottom row, Activation in VS during loss anticipation in control and COA groups and result of independent-sample $t$ test of control $>$ COA. Control and COA group activations are displayed at a threshold of $p<0.05$ FDR-corrected. Coordinates of coronal slice displayed were selected based on the peak voxel within the VS in which control $>$ COA. Color bars represent $T$ scores.

Table 3. Brain activation during anticipation of monetary loss versus no incentive for controls and $\mathrm{COAs}$

\begin{tabular}{llrrrll}
\hline \multirow{2}{*}{ Brain region } & \multicolumn{3}{l}{ MNI coordinates } & & & \\
\cline { 2 - 7 } & Left/right & $X$ & $y$ & $z$ & Peak $t$ value & $p$ (FDR-corrected) \\
\hline Controls & & & & & & \\
VS & Right & 12 & 8 & -6 & 5.78 & 0.002 \\
& Left & -10 & 6 & -6 & 5.46 & 0.003 \\
Thalamus & Mesial & 0 & -6 & -4 & 4.87 & 0.007 \\
$\quad$ Caudate & Left & -8 & 6 & 16 & 3.47 & 0.02 \\
COA & & & & & & \\
VS & Right & 12 & 16 & -12 & 5.04 & 0.006 \\
& Left & -6 & 8 & -4 & 4.31 & 0.02 \\
Thalamus & Mesial & 2 & -8 & 6 & 5.32 & 0.005 \\
Caudate & Left & -18 & -10 & 24 & 5.11 & 0.005 \\
MFG- orbital & Right & 20 & 48 & -20 & 4.65 & 0.01 \\
Insula & Right & 38 & 26 & 2 & 3.72 & 0.03 \\
\hline
\end{tabular}

with a grouping on alcohol use that captured not only consumption quantity but also other factors important to AUD risk, such as binge drinking and alcohol-related problems. Substance use information for each subgroup is summarized in Table 5. In the model for NAcc reward anticipation, there were significant main effects of family history (control $>\mathrm{COA} ; F_{(1,36)}=6.5, p=0.02$ ) and risky alcohol use (high-risk drinkers $>$ low-risk drinkers; $\left.F_{(1,36)}=5.2, p=0.03\right)$. There was also a significant interaction between family history and alcohol use $\left(F_{(1,36)}=4.4, p=0.04\right.$; Fig. $4 \mathrm{~B}$ ). Post hoc comparisons revealed a significant effect of risky drinking in COAs (low-risk-drinking COAs $<$ high-riskdrinking COAs; $p=0.003$ ) but not in controls and a significant effect of family history in low-risk drinkers (low-risk-drinking COAs $<$ low-risk-drinking controls; $p=0.008$ ) but not in highrisk drinkers.

In the model for loss anticipation, there were no main effects of family history or risky alcohol use $(p>0.1)$. There was again a significant interaction between family history and risky alcohol use $\left(F_{(1,36)}=4.5, p=0.04\right.$; Fig. $\left.4 B\right)$. Post hoc comparisons revealed a significant effect of risky drinking in COAs (low-riskdrinking COAs $<$ high-risk-drinking COAs; $p=0.009$ ) but not in controls and a trend effect of family history in low-risk drinkers (low-risk-drinking COAs $<$ low-risk-drinking controls; $p=$ $0.06)$ but not in high-risk drinkers.

Compared with high-risk-drinking COAs, low-risk-drinking COAs also had lower externalizing problems (low-risk-drinking COAs $T$ score $=39.3 \pm 5.0$; high-risk-drinking COAs $T$ score $=$ $50.4 \pm 8.1 ; t=3.3, p=0.004)$, whereas there were no differences between any other groups (all $p>0.18$ ). Finally, there was no difference between low-risk and high-risk COA drinkers in family severity index (low-risk-drinking COAs, $3.1 \pm 0.9$; high-riskdrinking COAs, $2.8 \pm 1.5 ; t=0.41, p>0.69)$.

\section{Discussion}

Modulation of the mesolimbic reward circuitry has been postulated as an important substrate underlying the development of alcoholism and other drug addiction (Blum et al., 2000; Robinson and Berridge, 2008). The current study examined the hypothesis that COAs show different reward circuitry responding beyond confounding effects of substance use, which may underlie their increased AUD risk. There were two main findings. First, blunted NAcc incentive response was only observed in COAs who had low alcohol use and related problems. Second, a link between early externalizing risk, NAcc responding, and current and lifetime alcohol consumption was observed in COAs but not controls.

A modified MID task was used to probe reward circuitry response during anticipation period between cue presentation and motor responding. Although both groups showed expected VS activation during anticipation of reward and loss avoidance, COAs demonstrated less right NAcc activation compared with controls. Because there were no behavioral differences between the groups as a result of individually calibrated task difficulty, this activation difference reflects differing responsivity of the saliency-reward system. This blunted motivational response, however, was not supportive of the reward-deficiency syndrome hypothesis of addiction vulnerability (Blum et al., 2000). Additional analyses revealed a significant family history $\times$ risky alcohol use interaction in which reduced NAcc responding was only observed in COAs who had been low drinkers, never binged, and experienced few alcohol-related prob- 
Table 4. Correlations between NAcc activation, drinks per week, lifetime drinking volume, and externalizing problems for total group, control, and COA groups separately

\begin{tabular}{|c|c|c|c|c|c|c|c|c|}
\hline & \multicolumn{8}{|c|}{ NAcc activation to } \\
\hline & \multicolumn{2}{|l|}{ Total } & \multicolumn{2}{|l|}{ Control } & \multicolumn{2}{|l|}{$\mathrm{COA}$} & \multicolumn{2}{|c|}{ Control versus $\mathrm{COA}^{a}$} \\
\hline & Reward & Loss & Reward & Loss & Reward & Loss & Reward & Loss \\
\hline Drinks per week & $0.33,0.04$ & $0.31,0.06$ & $-0.05,0.84$ & $-0.04,0.87$ & $0.71,<0.001^{b}$ & $0.61,0.004^{b}$ & $-2.7,0.007$ & $-2.2,0.03$ \\
\hline Lifetime drink volume & $0.17,0.29$ & $0.22,0.17$ & $-0.15,0.53$ & $-0.24,0.31$ & $0.48,0.04$ & $0.60,0.007$ & $-1.9,0.05$ & $-2.7,0.008$ \\
\hline Externalizing $T$ scores ages $12-14$ years & $-0.09,0.58$ & $-0.01,0.97$ & $-0.62,0.004^{b}$ & $-0.44,0.05$ & $0.60,0.005^{b}$ & $0.63,0.003^{b}$ & $-4.2,<0.001^{b}$ & $-3.5,<0.001^{b}$ \\
\hline \multicolumn{9}{|l|}{ Externalizing $T$ scores ages $12-14$ years } \\
\hline Drinks per week & \multirow{2}{*}{\multicolumn{2}{|c|}{$\begin{array}{c}0.49,0.001^{b} \\
0.55<001^{b}\end{array}$}} & \multicolumn{2}{|c|}{$0.42,0.07$} & \multicolumn{2}{|c|}{$0.60,0.005^{b}$} & \multicolumn{2}{|c|}{$-0.76,0.45$} \\
\hline Lifetime drink volume & & & \multicolumn{2}{|c|}{$0.18,0.46$} & \multicolumn{2}{|c|}{$0.77,<0.001^{b}$} & \multicolumn{2}{|c|}{$-2.4,0.02$} \\
\hline
\end{tabular}

${ }^{a}$ Results of Fisher's $Z$ transformation for testing differences between correlation coefficients.

${ }^{b}$ Survives correction for multiple comparisons $(0.05 / 8=p<0.0063)$.

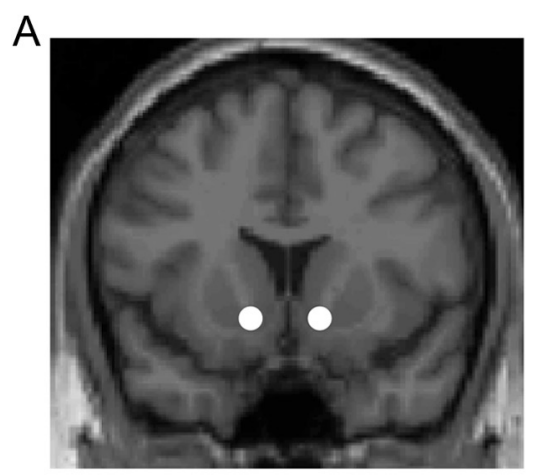

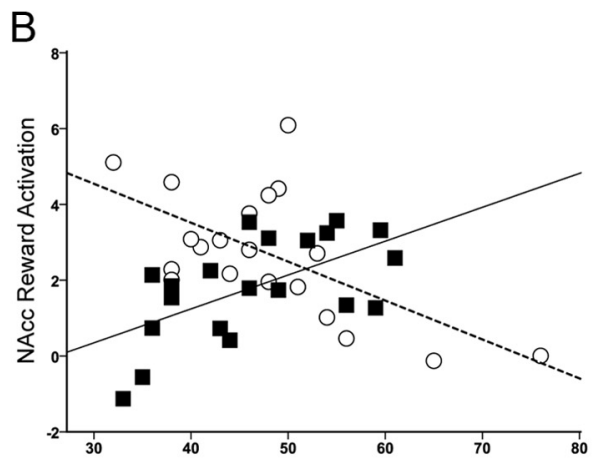

Externalizing Behavior Score Ages 12-14 (T-Scores)

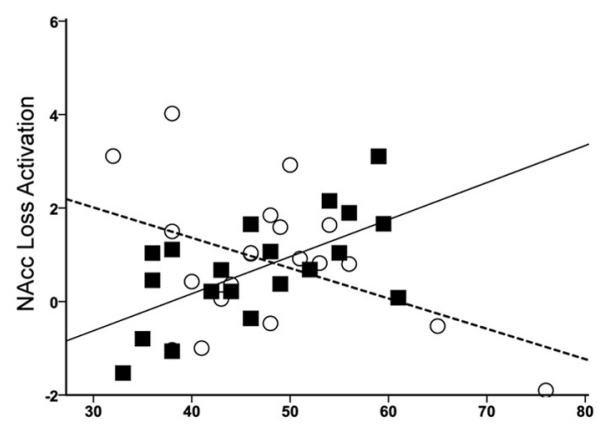

Externalizing Behavior Score Ages 12-14 (T-Scores)

Figure 3. A, Location of 5-mm-diameter spherical NAcc VOI mask at the ventromesial intersection of caudate and putamen ( $y=13)$. B, Correlation between effect size of NAcc activation during reward and loss anticipation and externalizing behavior score at ages 12-14 years in controls (white circles, dashed regression line) and COAs (black squares, solid regression line).

Table 5. Means (SDs) of substance use characteristics for subgroups in family history $\times$ risky alcohol use ANOVA

\begin{tabular}{|c|c|c|c|c|}
\hline & \multicolumn{2}{|l|}{ Control } & \multicolumn{2}{|l|}{$\mathrm{COA}$} \\
\hline & $\begin{array}{l}\text { Low-risk } \\
\text { drinker }\end{array}$ & $\begin{array}{l}\text { High-risk } \\
\text { drinker }\end{array}$ & $\begin{array}{l}\text { Low-risk } \\
\text { drinker }\end{array}$ & $\begin{array}{l}\text { High-risk } \\
\text { drinker }\end{array}$ \\
\hline $\mathrm{N}$ & 6 & 14 & 7 & 13 \\
\hline Males:Females & $2: 4$ & $6: 8$ & $3: 4$ & $5: 8$ \\
\hline Age (years) & $19.8(1.5)$ & $20.2(1.3)$ & $19.4(1.0)$ & $20.7(1.1)$ \\
\hline IQ & $116.7(6.6)$ & 111.7 & $110.0(15.7)$ & $111.4(11.0)$ \\
\hline Cigarettes per day (time of scan) & 0.0 & $2.0(5.6)$ & 0.0 & $3.5(6.1)$ \\
\hline Drinks per week (time of scan) & $0.5(0.8)$ & $8.4(7.4)$ & 0.0 & $7.9(7.8)$ \\
\hline Binge drinking days (past 6 months) & 0.0 & $54.1(58.9)$ & 0.0 & $73.8(84.1)$ \\
\hline Maximum drinks in $24 \mathrm{~h}$ & $0.7(1.0)$ & $12.0(8.3)$ & $0.3(0.8)$ & $14.5(7.8)$ \\
\hline Lifetime drink volume & $33.7(64.9)$ & $989.2(1170)$ & $2.1(5.7)$ & $1247.2(1065)$ \\
\hline Number of alcohol problems & $0.2(0.4)$ & $5.3(6.1)$ & $0.3(0.8)$ & $7.9(5.4)$ \\
\hline $\begin{array}{l}\text { Number of drug problems } \\
\text { Frequent marijuana use (past }\end{array}$ & 0.0 & $0.4(1.2)$ & $0.4(1.1)$ & $0.7(1.0)$ \\
\hline 12 months) & 0.0 & $2.5(3.3)$ & 0.0 & $3.4(3.2)$ \\
\hline Number of illicit drugs ever used & 0.0 & $1.9(2.7)$ & $0.1(0.4)$ & $1.9(1.2)$ \\
\hline
\end{tabular}

lems. High-risk-drinking COAs responded at the samelevel as controls, who showed no differential response across drinking risk levels. Furthermore, low-risk-drinking COAs who showed blunted NAcc response also had fewer precursive externalizing problems than high-riskdrinking COAs. The two COA groups did not differ in family severity index, indicating that these effects were not explained by the extent of liability within the nuclear family. Therefore, our results suggest that a lesser NAcc responsiveness to incentives may reflect a resilience mechanism, reducing risk for AUD development in COAs.

Early externalizing problems have consistently been identified as a risk factor for alcoholism (Cloninger et al., 1988; Zucker et al., 2008). Indeed, across our entire sample, externalizing prob- lems in early adolescence were correlated with both current and lifetime alcohol consumption. Our results further revealed a positive association among externalizing problems, current and lifetime alcohol use, and NAcc activation that was unique to COAs. Therefore, although control and COA groups were matched on early externalizing behavior and alcohol consumption, they differed in the relationship between these variables and NAcc response to incentive anticipation. It is of interest to note that, although externalizing behavior scores at time of scanning were missing from $20 \%$ of COAs and $5 \%$ of controls, analysis of this incomplete dataset revealed consistent findings of significant positive associations between current externalizing behavior, alcohol use, and NAcc activation in COAs but not controls. This suggests that a close association of behavioral risk, alcohol consumption, and motivational mechanisms may underlie addiction vulnerability in COAs. The absence of such systematic associations in controls may be a reflection of their lower overall AUD risk compared with COAs, which likely involves a more heterogeneous distribution of risk factors.

The current finding of a positive correlation between NAcc incentive activation and alcohol consumption in COAs may be interpreted in different ways. It may be driven by predisposed differences in reward circuitry reactivity. Alternatively, this may reflect a sensitization of reward circuitry after alcohol exposure, suggesting that COAs may be more susceptible to the modulating effects of alcohol on reward circuitry responding than controls. A recent study showed that healthy adults with a family history of alcoholism reported increased stimulating effects of alcohol and increased "wanting" and "liking" compared with controls with similar levels of current alcohol use (Söderpalm Gordh and Söderpalm, 2011). Prospective studies are needed to test these hypotheses. 
The finding of an opposite relationship between NAcc incentive activation and externalizing behavior in COAs versus controls was unexpected. However, Galvan et al. (2007) demonstrated opposing correlations between NAcc activation to reward and anticipated positive and negative consequences of risk-taking. Specifically, anticipated positive consequences correlated positively with NAcc activity, whereas anticipated negative consequences correlated negatively. Therefore, the opposite correlations found here between control and COA groups may relate to differences in their expectations about the outcomes of their externalizing behavior. This hypothesis requires additional exploration in future studies that include measures of the anticipated outcomes of behaviors.

The present findings also raise the important issue of individual variability of AUD risk in COAs and its relations to reward circuitry response. Transmission of familial liability factors can occur via multiple genetic effects (Goldman et al., 2005), as well as environmental effects associated with alcoholic households (Zucker et al., 2008). It has been shown that parental AUD history moderates the effect of dopamine transporter (DAT1) gene 10-repeat allele on increased alcohol problems in males, with the effect present only in those with an alcoholic father (Vaske et al., 2009). Furthermore, a recent study found a positive association between VS activation to reward anticipation and trait reward sensitivity in homozygote carriers of DAT110-repeat allele but not in other allele carriers (Hahn et al., 2011). These studies, along with the current findings, illustrate a complex interplay of genetic and environmental effects, reward-related personality traits, and reward circuitry functioning in relation to differential transfer of familial risk. Although it is beyond the scope of the current study, this will be important to investigate in future studies.

The direction of the present findings differs from that observed in adult alcoholics. Detoxified alcoholics showed reduced activation in the VS during anticipation of monetary incentives but increased activation to alcohol cues that was further associated with increased alcohol craving (Wrase et al., 2007). Past drug exposure has been shown to modulate subsequent motivational response in animal and human models (Wyvell and Berridge, 2001; Boileau et al., 2006; Nocjar and Panksepp, 2007). The findings in older alcoholics may therefore suggest a bias in reward system responsivity toward alcohol-related stimuli after continued alcohol use. More recently, an association between VS activation to incentive anticipation and self-reported impulsivity has been found in detoxified alcoholics but not in controls (Beck et al., 2009). This supports the hypothesis that the relationship between VS responding to incentive anticipation and behavioral risk may differ for those vulnerable to addiction.

In a sample of 12- to 16-year-old COAs and controls, Bjork et al. (2008b) found no difference in monetary incentive response between the groups. The difference from current findings may be attributable to the developmental period at which subjects were investigated. An emerging literature supports developmental changes in reward circuitry reactivity through the adolescent years. VS response to reward anticipation was shown to increase from adolescence to adulthood (Bjork et al., 2004, 2010). A developmental effect has also been observed in the association between NAcc re-

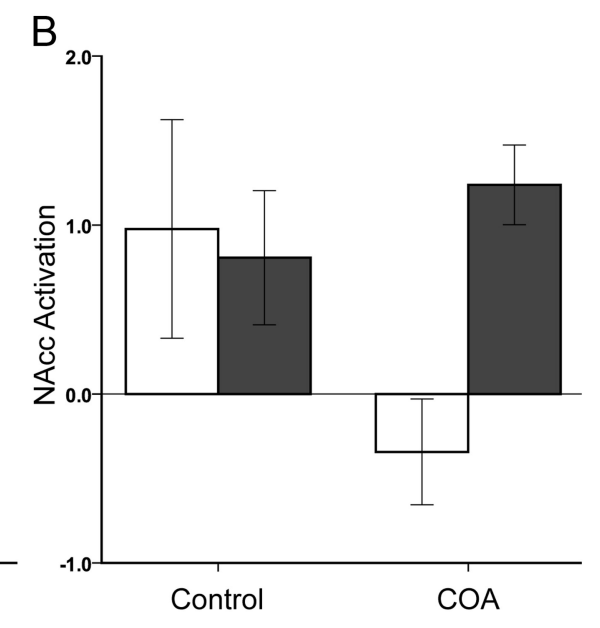

COA

Control

COA

Figure 4. Significant family history $\times$ risky alcohol use interaction for NAcc reward activation $(\boldsymbol{A})$ and NAcc loss activation $(\boldsymbol{B})$. White bars represent low-risk drinkers, and gray bars represent high-risk drinkers.

sponse during monetary reward anticipation and expectation of positive or negative consequence of risk-taking behavior (Galvan et al., 2007). Specifically, anticipated positive consequences were associated with more NAcc activation and greater likelihood of engaging in risky behavior in adults, whereas in children an association was found between anticipated negative consequences, lesser NAcc activation, and lesser likelihood of engaging in risky behavior. Associations in both directions were found in adolescents, suggesting a developmental shift in anticipation of consequences and related modulation of NAcc responsivity during this interval. The differences in NAcc responses found in the present study may not have emerged until late adolescence/early adulthood-a critical period in development when alcohol use and AUD prevalence reach their highest levels (Grant et al., 2004; Substance Abuse and Mental Health Services Administration, 2006). Prospective longitudinal studies are needed to investigate this possibility.

A limitation of this study is its relatively small sample size ( $n=$ 40 ), based on a priori selection of subjects from a larger sample to allow for accurate matching. A larger sample would allow for more accurate characterization of associations within individual groups. In addition, the current study focused on early externalizing problems (age 12-14 years) as a precursive behavioral measure before significant drinking and drug use. Future studies will be necessary to determine how current levels of externalizing problems and incentive responding may be linked in relation to risk and resilience.

The present study demonstrates a close association between NAcc activation to incentive anticipation, precursive behavioral risk, and alcohol consumption that is unique to COAs. The differences in correlations, along with a significant interaction between family history and alcohol use, show that the relationship between reward circuitry response and AUD risks differs between COAs and controls. The consistent findings across reward and loss anticipation is of additional significance because growing evidence suggests that motivational salience rather than valence/ hedonic effects is a key substrate in addiction development (Robinson and Berridge, 2008). Together, our findings suggest a multilevel dynamic process whereby a blunted NAcc response to incentive anticipation is related to lower precursive behavioral risk and is further associated with lesser alcohol use and related problems in COAs, potentially reflecting a resilience mechanism. Future prospective studies with larger groups of resilient and at-risk COAs and controls will help to confirm and expand these initial observations. 


\section{References}

Achenbach TM (1991) Manual for the youth self-report form and 1991 profile. Burlington, VT: University Associates in Psychiatry.

Beck A, Schlagenhauf F, Wüstenberg T, Hein J, Kienast T, Kahnt T, Schmack K, Hägele C, Knutson B, Heinz A, Wrase J (2009) Ventral striatal activation during reward anticipation correlates with impulsivity in alcoholics. Biol Psychiatry 66:734-742.

Bjork JM, Knutson B, Fong GW, Caggiano DM, Bennett SM, Hommer DW (2004) Incentive-elicited brain activation in adolescents: similarities and differences from young adults. J Neurosci 24:1793-1802.

Bjork JM, Smith AR, Hommer DW (2008a) Striatal sensitivity to reward deliveries and omissions in substance dependent patients. Neuroimage 42:1609-1621.

Bjork JM, Knutson B, Hommer DW (2008b) Incentive-elicited striatal activation in adolescent children of alcoholics. Addiction 103:1308-1319.

Bjork JM, Smith AR, Chen G, Hommer DW (2010) Adolescents, adults and rewards: comparing motivational neurocircuitry recruitment using fMRI. PLoS One 5:e11440.

Blum K, Braverman ER, Holder JM, Lubar JF, Monastra VJ, Miller D, Lubar JO, Chen TJ, Comings DE (2000) Reward deficiency syndrome: a biogenetic model for the diagnosis and treatment of impulsive, addictive, and compulsive behaviors. J Psychoactive Drugs 32 [Suppl i-iv]:1-112.

Boileau I, Dagher A, Leyton M, Gunn RN, Baker GB, Diksic M, Benkelfat C (2006) Modeling sensitization to stimulants in humans: an $\left[{ }^{11} \mathrm{C}\right]$ raclopride/positron emission tomography study in healthy men. Arch Gen Psychiatry 63:1386-1395.

Brett M, Anton JL, Valabregue R, Poline JB (2002) Region of interest analysis using an SPM toolbox. In: Eighth International Conference on Functional Mapping of the Human Brain, Sendai, Japan, June 2-6.

Carter RM, Macinnes JJ, Huettel SA, Adcock RA (2009) Activation in the VTA and nucleus accumbens increases in anticipation of both gains and losses. Front Behav Neurosci 3:21.

Chassin L, Pitts SC, DeLucia C, Todd M (1999) A longitudinal study of children of alcoholics: predicting young adult substance use disorders, anxiety, and depression. J Abnorm Psychol 108:106-119.

Cloninger CR, Sigvardsson S, Bohman M (1988) Childhood personality predicts alcohol abuse in young adults. Alcohol Clin Exp Res 12:494-505.

Englund MM, Egeland B, Oliva EM, Collins WA (2008) Childhood and adolescent predictors of heavy drinking and alcohol use disorders in early adulthood: a longitudinal developmental analysis. Addiction 103 [Suppl 1]:23-35.

Fessler J, Lee S, Olafsson V, Shi H, Noll D (2005) Toeplitz-based iterative image reconstruction for MRI with correction for magnetic field inhomogeneity. IEEE Trans Signal Proc 53:3393-3402.

Galvan A, Hare T, Voss H, Glover G, Casey BJ (2007) Risk-taking and the adolescent brain: who is at risk? Dev Sci 10:F8-F14.

Genovese CR, Lazar NA, Nichols T (2002) Thresholding of statistical maps in functional neuroimaging using the false discovery rate. Neuroimage 15:870-878.

Glover GH, Law CS (2001) Spiral-in/out BOLD fMRI for increased SNR and reduced susceptibility artifacts. Magn Reson Med 46:515-522.

Goldman D, Oroszi G, Ducci F (2005) The genetics of addictions: uncovering the genes. Nat Rev Genet 6:521-532.

Grant BF, Dawson DA, Stinson FS, Chou SP, Dufour MC, Pickering RP (2004) The 12-month prevalence and trends in DSM-IV alcohol abuse and dependence: United States, 1991-1992 and 2001-2002. Drug Alcohol Depend 74:223-234.

Hahn T, Heinzel S, Dresler T, Plichta MM, Renner TJ, Markulin F, Jakob PM, Lesch KP, Fallgatter AJ (2011) Association between reward-related activation in the ventral striatum and trait reward sensitivity is moderated by dopamine transporter genotype. Hum Brain Mapp 32:1557-1565.

Harford TC, Grant BF, Yi HY, Chen CM (2005) Patterns of DSM-IV alcohol abuse and dependence criteria among adolescents and adults: results from the 2001 National Household Survey on Drug Abuse. Alcohol Clin Exp Res 29:810-828.

Hariri AR, Brown SM, Williamson DE, Flory JD, de Wit H, Manuck SB (2006) Preference for immediate over delayed rewards is associated with magnitude of ventral striatal activity. J Neurosci 26:13213-13217.

Jenkinson M, Bannister P, Brady M, Smith S (2002) Improved optimization for the robust and accurate linear registration and motion correction of brain images. Neuroimage 17:825-841.
Knutson B, Westdorp A, Kaiser E, Hommer D (2000) FMRI visualization of brain activity during a monetary incentive delay task. Neuroimage 12:20-27.

Knutson B, Adams CM, Fong GW, Hommer D (2001) Anticipation of increasing monetary reward selectively recruits nucleus accumbens. J Neurosci 21:RC159(1-5).

Kriegeskorte N, Simmons WK, Bellgowan PS, Baker CI (2009) Circular analysis in systems neuroscience: the dangers of double dipping. Nat Neurosci 12:535-540.

Lacadie CM, Fulbright RK, Rajeevan N, Constable RT, Papademetris X (2008) More accurate Talairach coordinates for neuroimaging using non-linear registration. Neuroimage 42:717-725.

Loukas A, Fitzgerald HE, Zucker RA, von Eye A (2001) Parental alcoholism and co-occurring antisocial behavior: prospective relationships to externalizing behavior problems in their young sons. J Abnorm Child Psychol 29:91-106.

McClure SM, Laibson DI, Loewenstein G, Cohen JD (2004) Separate neural systems value immediate and delayed monetary rewards. Science 306:503-507.

National Institute on Alcohol Abuse and Alcoholism (2000) Alcohol involvement over the life course. In: NIAAA, Tenth Special Report to the U.S. Congress on Alcohol and Health: highlights from current research, pp 28-53. Bethesda, MD: U.S. Department of Health and Human Services.

Nocjar C, Panksepp J (2007) Prior morphine experience induces long-term increases in social interest and in appetitive behavior for natural reward. Behav Brain Res 181:191-199.

Robbins TW, Everitt BJ (1999) Drug addiction: bad habits add up. Nature 398:567-570.

Robinson TE, Berridge KC (2008) Review. The incentive sensitization theory of addiction: some current issues. Philos Trans R Soc Lond B Biol Sci 363:3137-3146.

Russell M (1990) Prevalence of alcoholism among children of alcoholics. In: Children of alcoholics: critical perspectives (Windle M, Searles J, eds), pp 9-38. New York: Guilford.

Sher KJ, Walitzer KS, Wood PK, Brent EE (1991) Characteristics of children of alcoholics: putative risk factors, substance use and abuse, and psychopathology. J Abnorm Psychol 100:427-448.

Söderpalm Gordh AH, Söderpalm B (2011) Healthy subjects with a family history of alcoholism show increased stimulative subjective effects of alcohol. Alcohol Clin Exp Res 35:1426-1434.

Sokol RJ, Clarren SK (1989) Guidelines for use of terminology describing the impact of prenatal alcohol on the offspring. Alcohol Clin Exp Res 13:597-598.

Substance Abuse and Mental Health Services Administration (2006) Results from the 2005 National Survey on Drug Use and Health: National Findings. NSDUH Series H-30, DHHS Publication SMA 06-4194. Rockville MD: SAMHSA, Office of Applied Studies.

Sutton BP, Noll DC, Fessler JA (2003) Fast, iterative image reconstruction for MRI in the presence of field inhomogeneities. IEEE Trans Med Imaging 22:178-188.

Vaske J, Beaver KM, Wright JP, Boisvert D, Schnupp R (2009) An interaction between DAT1 and having an alcoholic father predicts serious alcohol problems in a sample of males. Drug Alcohol Depend 104:17-22.

Wrase J, Schlagenhauf F, Kienast T, Wüstenberg T, Bermpohl F, Kahnt T, Beck A, Ströhle A, Juckel G, Knutson B, Heinz A (2007) Dysfunction of reward processing correlates with alcohol craving in detoxified alcoholics. Neuroimage 35:787-794.

Wyvell CL, Berridge KC (2001) Incentive sensitization by previous amphetamine exposure: increased cue-triggered "wanting" for sucrose reward. J Neurosci 21:7831-7840.

Zucker RA (1991) Scaling the developmental momentum of alcoholic process via the lifetime alcohol problems score. Alcohol Alcohol Suppl 1:505-510.

Zucker RA, Fitzgerald HE, Refior SK, Puttler LI, Pallas DM, Ellis DA (2000) The clinical and social ecology of childhood for children of alcoholics: description of a study and implications for a differentiated social policy. In: Children of addiction: research, health and policy issues (Fitzgerald HE, Lester BM, Zucker RA, eds), pp 109-141. New York: Routledge Falmer.

Zucker RA, Donovan JE, Masten AS, Mattson ME, Moss HB (2008) Early developmental processes and the continuity of risk for underage drinking and problem drinking. Pediatrics 121 [Suppl 4]:S252-S272.

Zucker R, Ellis D, Fitzgerald H, Bingham C, Sanford K (1996) Other evidence for at least two alcoholisms. II. Life course variation in antisociality and heterogeneity of alcoholic outcome. Dev Psychopathol 8:831-848. 